\title{
Nefrocalcinosi in età pediatrica
}

\author{
C. La Scola, F. Mencarelli, A. Pasini, S. Visentin, G. Montini
}

Struttura di Nefrologia Dialisi Pediatrica dell'Azienda Ospedaliero-Universitaria di Bologna, Policlinico S. Orsola-Malpighi, Bologna

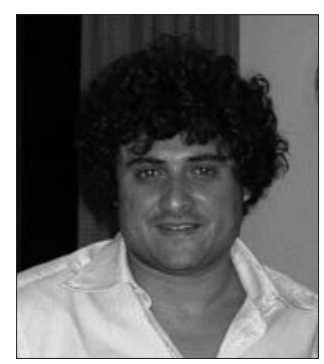

C. La Scola

\section{Introduzione}

La Nefrocalcinosi (NC) è un quadro morfologico renale caratterizzato da un aumentato deposito di calcio a livello del parenchima renale. Tale condizione si associa solitamente ad alcune condizioni cliniche caratterizzate o da ipercalciuria e/o da ipercalcemia e ne rappresenta in alcuni casi il primo riscontro clinico.

La reale dimensione del problema non è facile da stabilire e spesso tale condizione rimane misconosciuta. Questo è dovuto, soprattutto, alla soggettività della tecnica radiologica principe nella diagnosi di $\mathrm{NC}$ ovvero l'ecografia. Inoltre, dal punto di vista anatomopatologico, è frequente durante l'allestimento dei preparati istologici la perdita di cristalli di calcio dal tubulo renale.

Oggi, comunque, con l'affinarsi delle tecniche ecografiche, tale condizione clinica viene diagnosticata in un crescente numero di soggetti ed inoltre è in aumento la conoscenza delle patologie che possono associarsi a tale condizione clinica (Tab. I).

\section{Cause di nefrocalcinosi}

Le NC può essere dovuta a diverse cause (Tab. I): esogene, ereditarie oppure ad una miscellanea di altre patologie che non sempre riguardano il rene. Infine, una forma particolare e specifica di NC è quella del pretermine; tale forma verrà discussa nel corso di questa trattazione.

In uno studio retrospettivo italiano condotto da Ammenti et al (1) su una popolazione di 41 bambini la causa principale di NC era rappresentata dalle pato- logie del tubulo (41\%). La tubulopatia maggiormente associata alla $\mathrm{NC}$ risultava essere l'acidosi tubulare distale (17\% dei casi totali). Da sottolineare che in un $24 \%$ di casi la reale causa di NC rimaneva sconosciuta. Le altre cause di NC erano rappresentate da: intossicazione da vitamina D nel 10\% dei casi, iperossaluria nel 7\% dei casi, dall'ipercalciuria idiopatica nel $5 \%$ dei casi ed infine in un $12 \%$ dei casi da cause più rare (Sindrome di Down, ipercalcemia con ipercalciuria, sindrome di Patau, ipoplasia surrenalica, miopatia miotubulare X-linked).

Oltre alla casistica italiana, altri due studi retrospettivi hanno indagato le cause e il follow-up della NC.

Il primo, in ordine cronologico, è uno studio su una popolazione di 152 bambini condotto in Germania da Rönnefarth et al (2). In questo studio il $35 \%$ dei casi di NC era associato ad ipercalciuria mentre nel $23 \%$ dei casi la NC era dovuta a tubulopatie ereditarie. Le forme senza causa erano il $6 \%$. Da questo studio sono stati esclusi i pazienti con iperossaluria primitiva. Il secondo studio è stato condotto da Mantan et al (3) su un gruppo di 40 bambini indiani. In questa popolazione, la causa principale era rappresentata nel 50\% dei casi dall'acidosi tubulare distale seguita dall'ipercalciuria idiopatica (7.5\%) e dall'iperossaluria (7.5\%). Le forme da causa sconosciuta ammontavano al $12.5 \%$.

\section{Diagnosi di nefrocalcinosi}

La diagnosi di nefrocalcinosi è prettamente radiologica. Le metodiche radiologiche più affidabili per la diagnosi sono l'ecografia e la tomografia computerizzata (TC) anche se la prima è la tecnica più sensibile e affidabile per lo screening e il grading della NC (4-6). 
TABELLA I - CAUSE DI NEFROCALCINOSI

$\begin{array}{lll}\text { Forme } & \text { Cause }\end{array}$

Nefrocalcinosi del prematuro

Nefrocalcinosi da cause esogene

Nefrocalcinosi da malattie ereditarie

Miscellanea

Dal punto di vista ecografico possiamo distinguere forme midollari, corticali o globali (Tab. II).

Il pattern ecografico più frequente in età pediatrica è quello midollare presente dall' 85 al 95\% dei casi che si caratterizza per l'iperecogenicità della midollare; più raro è il riscontro delle forme corticali o globali (1). La forma midollare può essere classificata a seconda dell'entità dell'ecogenicità in tre gradi (5):

I grado $=$ Modico incremento dell'ecogenicità attorno al bordo delle piramidi midollari.

II grado = Incremento più diffuso dell'ecogenicità a livello delle piramidi midollari

III grado = Omogenea ecogenicità a livello delle piramidi midollari.
Farmaci: furosemide, desametasone

Intossicazione da etilen-glicole

Intossicazione da vitamina $\mathrm{D}$ o $\mathrm{A}$

Nutrizione parenterale

Terapia con ormone adrenocorticotropo

Anemia falciforme

Iperossaluria primaria

Ipomagnesemia familiare con ipercalciuria e NC

Malattia di Wilson

Rachitismo ipofosfatemico con ipercalciuria

Rene a spugna midollare

Sindrome di Bartter

Sindrome di Dent

Sindrome di Lesh-Nyhan

Sindrome di Lowe

Sindrome di William

Tirosinemia

Acidosi tubulare distale

Insufficienza surrenalica

Ipercalcemia cronica

Ipercalcemia idiopatica

Iperparatiroidismo

Ipertiroidismo

Ipotiroidismo

Metastasi ossee

Necrosi lipoidea

Neoplasie maligne

Sarcoidosi ed altre malattie granulomatose

Sindrome di Cushing

\section{Anatomia e istologia delle NC}

Dal punto di vista anatomopatologico distinguiamo due forme: la microscopica e la macroscopica (7). La forma microscopica è rilevabile solo per la presenza al microscopio ottico di cristalli di fosfato di calcio o ossalato di calcio. La forma macroscopica di contro si caratterizza per la presenza di grandi aeree di calcificazione facilmente individuabili all'ecografia.

I sali di calcio possono depositarsi in diverse zone del parenchima renale.

I siti maggiormente coinvolti sono:

- Le cellule tubulari renali; 
TABELLA II - CAUSE DI NEFROCALCINOSI IN BASE ALLA FORMA ECOGRAFICA

\begin{tabular}{|c|c|}
\hline Nefrocalcinosi & Cause \\
\hline Midollare & $\begin{array}{l}\text { Acidosi tubulare distale } \\
\text { Anemia falciforme } \\
\text { Farmaci: furosemide, desametasone } \\
\text { Insufficienza surrenalica } \\
\text { Intossicazione da vitamina D o A } \\
\text { Ipercalcemia idiopatica } \\
\text { Iperossaluria } \\
\text { Iperparatiroidismo } \\
\text { Ipertiroidismo } \\
\text { Ipotiroidismo } \\
\text { Malattia di Wilson } \\
\text { Metastasi ossee } \\
\text { Necrosi lipoidea } \\
\text { Neoplasie maligne } \\
\text { Nutizione parenterale } \\
\text { Prematurità < 32 settimane } \\
\text { Rene a spugna midollare } \\
\text { Sarcoidosi ed altre malattie granulomatose } \\
\text { Sindrome di Bartter } \\
\text { Sindrome di Cushing } \\
\text { Sindrome di Lesh-Nyhan } \\
\text { Sindrome di Lowe } \\
\text { Sindrome di William } \\
\text { Terapia con ormone adrenocorticotropo } \\
\text { Tirosinemia }\end{array}$ \\
\hline
\end{tabular}

Corticale

Anemia Falciforme

Intossicazione da etilen-glicole

Ipeossularia primaria

Ipercalcemia cronica

Diagnosi

Differenziale
- L’interstizio renale;

- L'interno del lume tubulare (in questo caso l'ostruzione può causare una tubulopatia che potrebbe evolvere verso l'insufficienza renale cronica).

\section{Clinica}

Dal punto di vista clinico la NC si correla, il più delle volte, alla sintomatologia della patologia di base, infatti di per sé la NC non dà segni tangibili della sua presenza. Sia nello studio clinico italiano condotto da Ammenti et al che nello studio tedesco condotto da Rönnefarth et al il segno clinico maggiormente associato alla $\mathrm{NC}$ era il ritardo di crescita (rispettivamente nel 41 e 46\% delle casistiche), che però va interpretato come una conseguenza della malattia associata e non della NC stessa (1, 2). Altri segni clinici descritti in entrambi gli studi erano il ritardo psicomotorio, le infezioni delle vie urinarie, la nefrolitiasi, il dolore addominale ricorrente e disfunzione vescicale.

I quadri clinici che comunque possono associarsi con la NC all'esordio sono (8):

- La colica renale o l'ematuria (micro o macroscopica);

- Le infezioni delle vie urinarie;

- La poliuria e polidipsia;

- Lipertensione arteriosa (rara);

- La proteinuria di solito in range non nefrosico $(<500$ $\mathrm{mg} / 24 \mathrm{~h})$;

- La piuria microscopica a causa della risposta infiammatoria cronica alle calcificazioni midollari;

- La perdita di sali e acidosi metabolica per alterazioni tubulari;

- L’insufficienza renale cronica;

- Altri sintomi riferibili alle patologie di base.

\section{Work-up}

In caso di riscontro di $\mathrm{NC}$, nel momento in cui non se ne conoscesse la causa, è utile eseguire alcuni esami di laboratorio per l'inquadramento diagnostico (9):

- Dosaggio sierico di calcio, fosfato e albumina. Tali dosaggi sono necessari per stabilire se la NC sia associata ad ipercalcemia. Il dosaggio dell'albumina è importante in quanto nel caso di ipoalbuminemia la reale concentrazione di calcio potrebbe essere sottostimata. Se riscontriamo livelli bassi di fosfato dobbiamo sospettare una forma di iperparatiroidismo primitivo con funzionalità renale normale o una forma di rachitismo ipofosfatemico dovuto alla perdita urinaria di fosforo. Di contro il fosforo è elevato nelle forme di NC associate a insufficienza renale cronica.

- Dosaggio contemporaneo degli elettroliti sierici, dell'urea e della creatinina e del $\mathrm{pH}$ ematico e urinario. Il potassio potrebbe essere ridotto qualora la $\mathrm{NC}$ si associ a patologie come la sindrome di Bartter, la acidosi tubulare distale, l'iperaldosteronismo primitivo 

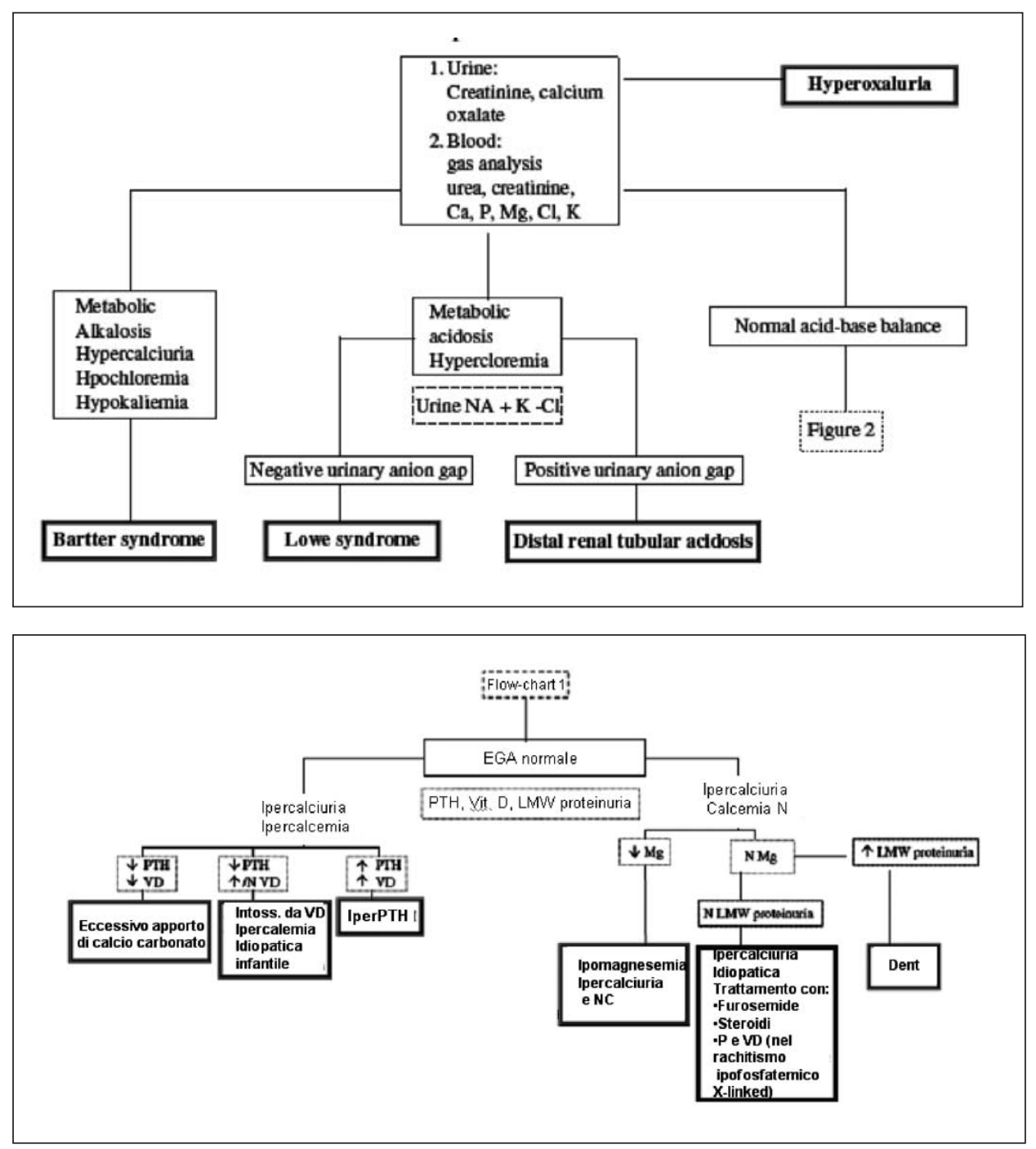

Fig. 1 - Flow-chart II: algoritmo diagnostico per la nefrocacalcinosi in età pediatrica modificato da Ammenti A, Pelizzoni A, Cecconi $M$, Molinari PP, Montini G. Nephrocalcinosis in children: a retrospective multi-centre study.Acta Paediatr 2009 Jul 1. [Epub ahead of print].

Fig. 2 - Flow-chart II: algoritmo diagnostico per la nefrocacalcinosi in età pediatrica modificato da Ammenti A, Pelizzoni A, Cecconi M, Molinari PP, Montini G. Nephrocalcinosis in children: a retrospective multi-centre study.Acta Paediatr. 2009 Jul 1. [Epub ahead of print]. o la sindrome di Liddle.

- L'esame delle urine con analisi microscopica del sedimento. Tale esame permette di escludere forme infettive croniche delle vie urinarie; inoltre il riscontro di $\mathrm{pH}$ urinario basso è suggestivo di acidosi tubulare distale.

- Dosaggio sulla raccolta delle urine delle 24 ore di calciuria, ossaluria citraturia, uricuria e proteinuria con determinazione simultanea di creatinina urinaria. Una eccessiva eliminazione di calcio con le urine potrebbe essere osservato in pazienti con ipercalciuria idiopatica. L'incremento dell'ossaluria indica forme di NC dovute a iperossaluria primitiva o secondaria. I pazienti con NC generalmente presentano proteinuria di modesta entità solitamente di origine non glomerulare. La proteinuria in range nefrosico non è un riscontro atteso in questa patologia e qualora fosse presente andrebbe indagata.

- Dosaggio del PTH in caso di ipercalcemia o di insufficienza renale.
- Dosaggio del TSH in quanto i disordini tiroidei possono associarsi a NC.

- Dosaggio dei livelli urinari di magnesio utili per diagnosticare una eventuale patologia renale magnesio disperdente.

Per un migliore orientamento diagnostico vedi flowchart I e II.

\section{Prognosi}

La mortalità e morbilità della NC sono principalmente dovute alle patologie di base piuttosto che alla sola NC.

Dall'analisi degli studi retrospettivi condotti sulla popolazione italiana, tedesca e indiana rispettivamente da Ammenti et al, Rönnefarth et al e da Mantan et al si osserva un buon recupero della crescita staturo-ponderale e un basso rischio di evoluzione verso la IRC grave, una volta corrette le alterazioni legate alla causa che ha provocato la NC. 
In particolare nello studio italiano (1), dopo un follow-up mediano di 4 anni e 5 mesi su 28 dei 41 pazienti studiati (range da $1 \mathrm{a} 23$ anni) si è assistito a: 1) un miglioramento della crescita in altezza da un valore mediano di -2.2 deviazioni standard (SD) a un valore di - 1.0 (in nessuno dei casi è stato utilizzato $\mathrm{GH}$ ); 2) riduzione del filtrato glomerulare solo in un paziente che di base era affetto da sindrome di Dent. Inoltre il grado di NC è peggiorato in $16(62 \%)$ pazienti, è rimasto stabile in $8(30 \%)$ ed è migliorato in 2 pazienti ( $8 \%)$.

Nello studio condotto su 152 bambini da Rönnefarth et al (2) dopo un follow-up mediano 4.1 anni si è assistito a: 1) un miglioramento della crescita corporea ( $41 \%$ con statura < di 2 SD al primo controllo vs $32 \%$ all'ultimo controllo); 2) una riduzione lieve della funzionalità renale (il $6 \%$ mostrava una filtrazione glomerulare $<50 \mathrm{~mL} /$ $\min / 1.73 \mathrm{~m}^{2}$ ).

Solo nello studio condotto su 40 bambini indiani con NC (3), dopo un follow-up mediano di 35 mesi (range 14240), si è assistito ad un significativo peggioramento della funzionalità renale da $82.0(42-114) \mathrm{mL} / \mathrm{min}$ per 1.73 $\mathrm{m}^{2}$ a $70.8(21.3-126.5) \mathrm{mL} / \mathrm{min}$ per $1.73 \mathrm{~m}^{2}(\mathrm{p}=0.001)$.

\section{Nefrocalcinosi del pretermine}

La NC del prematuro è stata descritta per la prima volta da Hufnagle nel 1982 in prematuri che venivano trattati a lungo con la furosemide (10).

La nefrocalcinosi nei prematuri è riportata a seconda delle casistiche dal 7 al 41\% dei nati pretermine con età gestazionale $<$ di 32 settimane o peso alla nascita inferiore a $1500 \mathrm{~g}(11-16)$.

La differente frequenza è dovuta da una parte alle diverse popolazioni studiate, dall'altra alla strumentazione ecografica a disposizione dell'operatore e alla soggettività della metodica stessa.

Anche nel pretermine la NC è causata dal deposito intratubulare di cristalli di ossalato di calcio o meno frequentemente di fosfato di calcio.

Nel pretermine, la NC ha una eziopatogenesi multifattoriale, essendo dovuta da una parte alla bassa età gestazionale e al basso peso e dall'altra ai quadri patologici che possono interessarlo come ad esempio il grave distress respiratorio (17).

Bisogna sottolineare inoltre che i reni dei bambini prematuri hanno nefroni relativamente ben sviluppati ma con una lunga ansa di Henle in cui la velocità delle urine è ridotta. Tale condizione favorisce la formazione di cristalli che depositandosi all'interno dei tubuli danno origine ad aggregati. A questo, inoltre, si aggiunge l'alterazione del bilancio tra i fattori promuoventi (aumentati) e fattori inibenti (ridotti) la formazione dei calcoli, quali:

1. L'ipercalciuria dovuta all'acidosi metabolica, all'utilizzo di farmaci (diuretici dell'ansa, glucocorticoidi, metilxantine e vitamina $\mathrm{D}$ ), alla nutrizione parenterale e all'alto apporto di calcio e al basso apporto di fosforo con la dieta;

2. L’iperossaluria;

3. Il sesso maschile;

4. La familiarità per calcoli;

5. L'assunzione di farmaci nefrotossici;

6. La bassa concentrazione di citrato urinario;

7. La bassa concentrazione di macromolecole inibenti la formazione di calcoli (osteopontina, nefrocalcina, proteine di Tamm Horsfall).

Dal punto di vista prognostico, diversi studi hanno analizzato in questa popolazione l'evoluzione e le conseguenze della $\mathrm{NC}$ a breve e lungo termine, soprattutto per quanto riguarda la persistenza, il rischio di sviluppare urolitiasi, ipertensione arteriosa, insufficienza renale, tubulopatia e ipercalciuria.

1) Per quanto riguarda la persistenza della nefrocalcinosi nel pretermine, in circa l'80-90\% dei bambini si osserva la risoluzione spontanea nel primo anno di vita. In uno studio osservazionale condotto da Schell-Feith et al (18) su 201 neonati pretermine la NC persisteva nel 34\% dei pazienti dopo 15 mesi, nel 15\% dopo 30 mesi e nel 10\% dopo $7.4( \pm 1.0)$ anni.

2) Il rischio di nefrolitiasi è basso; in uno studio prospettico condotto da Jones et al nessuno dei bambini studiati ha sviluppato urolitiasi durante il follow-up di due anni (19).

3) Per quanto riguarda il rischio di sviluppare ipertensione arteriosa si è visto che questo non è aumentato. In uno studio prospettico condotto da Kist-van Holthe et al su 74 ex-pretermine con età gestazionale $<$ di 32 settimane (42 con NC e 32 senza) all'età di $7.4( \pm 1.0)$ anni si è visto che la $\mathrm{NC}$ non rappresentava un fattore di rischio per lo sviluppo di ipertensione arteriosa né per la crescita renale (20). Da sottolineare che entrambe le popolazioni di pretermine avevano valori pressori più elevati rispetto al normale per età.

4) Anche per quanto riguarda la funzionalità renale diversi studi ne hanno dimostrato una normalità nel lungo termine $(21,22)$.

5) Per quanto riguarda la funzionalità tubulare gli studi effettuati hanno dimostrato una maggiore compromissione del tubulo distale rispetto al prossimale, soprattutto per quanto concerne l'acidificazione delle urine $(20,22)$.

6) Infine si è visto che ex pretermine con $\mathrm{NC}$ hanno un maggior rischio di sviluppare ipercalciuria nel lungo termine (20). 


\section{Conclusioni}

La NC può associarsi a vari quadri patologici nefrourologici e non. Infatti a volte la patologia di base non compete direttamente il rene ma colpisce altri distretti corporei (per esempio nelle affezioni della tiroide o delle paratiroidi) e quindi "la caccia" alla causa deve essere condotta anche "al di fuori del rene".

Bisogna ricordare che i prematuri sono una popolazione particolarmente a rischio di sviluppare la $\mathrm{NC}$ e che quindi la stessa deve essere ricercata mediante controlli ecografici di screening.

La prognosi della NC a medio termine è comunque buona per crescita corporea e funzione renale nel momento in cui venga identificata e trattata la pato- logia di base che l'ha causata. L'evoluzione negativa è, infatti, maggiormente legata alla malattia di fondo per cui questi pazienti dovranno essere seguiti a lungo termine anche al di là dell'età pediatrica.

\section{Indirizzo degli Autori: \\ Claudio La Scola, MD \\ Struttura di Nefrologia Dialisi Pediatrica \\ Azienda Ospedaliero-Universitaria di Bologna \\ Policlinico S. Orsola-Malpighi \\ Bologna, Italy \\ clasc@libero.it}

\section{Bibliografia}

1. Ammenti A, Pelizzoni A, Cecconi M, Molinari PP, Montini G. Nephrocalcinosis in children: a retrospective multicentre study. Acta Paediatr 2009 Jul 1. [Epub ahead of print].

2. Rönnefarth G, Misselwitz J, Members of APN. Nephrocalcinosis in children: a retrospective survey. Pediatr Nephrol 2000; 14: 1016-21.

3. Mantan M, Bagga A, Virdi VS, Menon S, Hari P. Etiology of nephrocalcinosis in children. Pediatr Nephrol 2007; 22: 829-33.

4. Alon U, Brewer WH, Chan JC. Nephrocalcinosis: detection by ultrasonography. Pediatrics 1983; 71(6): 970-3.

5. Dick PT, Shuckett BM, Tang B, Daneman A, Kooh SW. Observer reliability in grading nephrocalcinosis on ultrasound examinations in children. Pediatr Radiol 1999; 29(1): 68-72.

6. Schell-Feith EA, Holscher HC, Zonderland HM, Kist-Van Holthe JE, Conneman N, van Zwieten PH, et al. Ultrasonographic features of nephrocalcinosis in preterm neonates. Br J Radiol 2000; 73(875): 1185-91.

7. Evan AP, Lingeman J, Coe F, Shao Y, Miller N, Matlaga B et al. Renal histopathology of stone-forming patients with distal renal tubular acidosis. Kidney Int 2007; 71(8): 795801.

8. Khan AN, MacDonald S, Chandramohan M, Chandramohan H. Nephrocalcinosis. Disponibile su http://emedicine.medscape.com/article/379449 (accesso 20/08/2009).

9. Hoppe B, Kemper MJ. Diagnostic examination of the child with urolithiasis or nephrocalcinosis. Pediatr Nephrol 23 2008. [Epub ahead of print].

10. Hufnagle KG, Khan SN, Penn D, Cacciarelli A, Williams P. Renal calcifications: a complication of long-term furosemide therapy in preterm infants. Pediatrics 1982; 70(3): 360-3.

11. Jacinto JS, Modanlou HD, Crade M, Strauss AA, Bosu SK. Renal calcification incidence in very low birth weight infants. Pediatrics 1988; 81(1): 31-5.

12. Short A, Cooke RW. The incidence of renal calcification in preterm infants. Arch Dis Child 1991; 66(4 Spec No): 412-7.

13. Sheu JN, Chen CH, Lue KH, Chen JY, Tsau YK, Chen JH. Renal calcification in very low birth weight infants. Am J Nephrol 1993; 13(1): 6-11.

14. Saarela T, Vaarala A, Lanning P, Koivisto M. Incidence, ultrasonic patterns and resolution of nephrocalcinosis in very low birthweight infants. Acta Paediatr 1999; 88(6): 655-60.

15. Hoppe B, Duran I, Martin A, Kribs A, Benz-Bohm G, Michalk DV, Roth B. Nephrocalcinosis in preterm infants: a single center experience. Pediatr Nephrol 2002; 17(4): 264-8

16. Hein G, Richter D, Manz F, Weitzel D, Kalhoff H. Development of nephrocalcinosis in very low birth weight infants. Pediatr Nephrol 2004; 19(6): 616-20. Epub 2004 Mar 31.

17. Schell-Feith EA, Kist-van Holthe JE, van der Heijden AJ. Nephrocalcinosis in preterm neonates. Pediatr Ne- 
phrol 2008 Sep 17.

18. Schell-Feith EA, Kist-van Holthe JE, Zwieten PH, Zonderland M, Holscher HC, Swinkels DW, et al. Preterm neonates with nephrocalcinosis: natural course and renal function. Pediatr Nephrol 2003; 18(11): 1102-8. Epub 2003 Oct 2.

19. Jones CA, King S, Shaw NJ, Judd BA. Renal calcification in preterm infants: follow up at 4-5 years. Arch Dis Child Fetal Neonatal Ed 1997; 76(3): F185-9.

20. Kist-van Holthe JE, van Zwieten PH, Schell-Feith EA, Zonderland HM, Holscher HC, Wolterbeek R, et al. Is nephro- calcinosis in preterm neonates harmful for long-term blood pressure and renal function? Pediatrics 2007; 119(3): 468-75. Erratum in: Pediatrics 2008 Jan; 121(1): 227.

21. Saarela T, Lanning P, Koivisto M. Prematurity-associated nephrocalcinosis and kidney function in early childhood. Pediatr Nephrol 1999; 13(9): 886-90.

22. Porter E, McKie A, Beattie TJ, McColl JH, Aladangady N, Watt A, White MP. Neonatal nephrocalcinosis: long term follow up. Arch Dis Child Fetal Neonatal Ed. 2006; 91(5): F333-6. Epub 2006 May 16. Erratum in: Arch Dis Child Fetal Neonatal Ed. 2007 Jan; $92(1)$ : F78. 